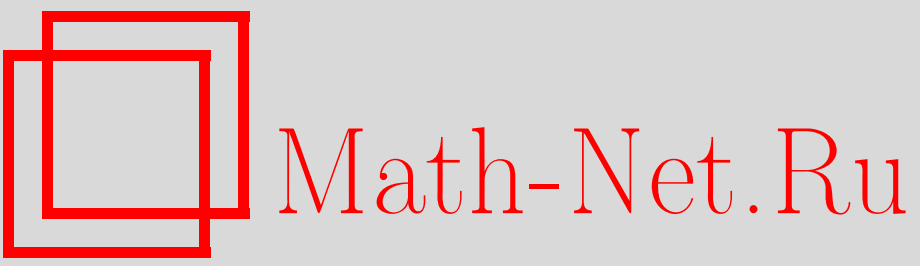

Г. А. Султанова, Об алгебрах Ли инфинитезимальных аффинных преобразований касательных расслоений, Итоги науки и техн. Сер. Соврем. мат. и ее прил. Темат. обз., 2020, том 180, 103-108

DOI: https://doi.org/10.36535/0233-6723-2020-180-103-108

Использование Общероссийского математического портала Math-Net.Ru подразумевает, что вы прочитали и согласны с пользовательским соглашением

http://www.mathnet.ru/rus/agreement

Параметры загрузки:

IP : 54.166 .219 .16

26 апреля 2023 г., 13:01:20 


\title{
ОБ АЛГЕБРАХ ЛИ ИНФИНИТЕЗИМАЛЬНЫХ АФФИННЫХ ПРЕОБРАЗОВАНИЙ КАСАТЕЛЬНЫХ РАССЛОЕНИЙ
}

\author{
(c) 2020 г. $\quad$ Г. А. СУЛТАНОВА
}

\begin{abstract}
АннотАция. В работе исследуются максимальные размерности алгебр Ли инфинитезимальных аффинных преобразований касательных расслоений со связностью полного лифта над пространствами полусимметрической связности.
\end{abstract}

Ключевые слова: касательное расслоение, инфинитезимальное аффинное преобразование, алгебра Ли, полусимметрическая связность.

\section{LIE ALGEBRAS OF INFINITESIMAL AFFINE TRANSFORMATIONS OF TANGENT BUNDLES}

\author{
(c) 2020 G. A. SULTANOVA
}

\begin{abstract}
In this paper, we examine maximal dimensions of Lie algebras of infinitesimal affine transformations of tangent bundles with a complete lift connection over spaces with a semisymmetric connection.
\end{abstract}

Keywords and phrases: tangent bundle, infinitesimal affine transform, Lie algebra, semisymmetric connection.

AMS Subject Classification: 53B05, 53A55

1. Введение. Касательные расслоения первого порядка $T M$ гладких многообразий возникли в середине 1930-х гг. прошлого столетия в связи с созданием теории расслоенных пространств. Первые результаты по теории касательных расслоений принадлежали А. Моримото, Ш. Сасаки, К. Яно, С. Исихара. Среди отечественных ученых касательные расслоения и их обобщения исследовали А. П. Широков, В. В. Вишневский, В. В. Шурыгин, Б. Н. Шапуков, Ф. И. Каган и другие.

Вопросами аффинных преобразований в касательных расслоениях занимались К. Яно, Ш. Кобаяси, К. Сато, Ш. Танно. Исследованию касательных расслоений и их обобщений, а также их автоморфизмов, снабженных различными геометрическими структурами, посвящены работы В. Г. Подольского, Х. Шадыева. В настоящее время автоморфизмы расслоений А. Вейля рассматриваются А. Я. Султановым.

В данной работе исследуются максимальные размерности алгебр Ли инфинитезимальных аффинных преобразований в касательных расслоениях, снабженных полным лифтом полусимметрической связности, заданной на многообразии.

2. Основные понятия. Пусть $M$-связное гладкое многообразие класса $C^{\infty}$ размерности $n$ $(n \geqslant 2), C^{\infty}(M)$ - алгебра вещественнозначных функций класса $C^{\infty}$ на $M$. 
Рассмотрим линейную связность $\nabla$, заданную на $M$. С этой связностью связаны два тензорных поля $T$ и $R$ кручения и кривизны, соответственно, определяемые тождествами

$$
\begin{gathered}
T(X, Y)=\nabla_{X} Y-\nabla_{Y} X-[X, Y], \\
R(X, Y) Z=\nabla_{X} \nabla_{Y} Z-\nabla_{Y} \nabla_{X} Z-\nabla_{[X, Y]} Z .
\end{gathered}
$$

Линейная связность $\nabla$ позволяет задавать на $M$ еще одну линейную связность $\hat{\nabla}$ условием (см. [2])

$$
\hat{\nabla}_{X} Y=\nabla_{Y} X+[X, Y]
$$

для произвольных векторных полей $X, Y \in \mathfrak{T}_{0}^{1}(M)$. Линейные связности $\hat{\nabla}$ и $\nabla$ удовлетворяют тождеству

$$
\hat{\nabla}_{X} Y=\nabla_{X} Y-T(X, Y) \text {. }
$$

Тензорные поля кручения $T$ и $\hat{T}$ связностей $\nabla$ и $\hat{\nabla}$ связаны между собой соотношением $\hat{T}+T=0$. Ясно, что если $T=0$, то $\hat{\nabla}=\nabla$. С помощью линейных связностей $\hat{\nabla}, \nabla$ определим линейную связность $\stackrel{0}{\nabla}$ на $M$ условием

$$
\stackrel{0}{\nabla}_{X} Y=\frac{1}{2}\left(\nabla_{X} Y+\hat{\nabla}_{X} Y\right)
$$

Прямые вычисления позволяют установить, что ее тензорное поле $\stackrel{0}{T}$ кручения равно нулю.

Линейную связность $\stackrel{0}{\nabla}$ принято называть сопутствующей связностью без кручения для связности $\nabla$ (см. [2]).

Пространство линейной связности с ненулевым тензорным полем кручения $T$ обозначим, следуя И. П. Егорову, через $\Lambda_{n}$.

Определение 1 (см. [3]). Пространство $\Lambda_{n}$ называется пространством полусимметрической линейной связности, если тензорное поле кручения $T$ удовлетворяет условию

$$
T=\varphi \otimes I-I \otimes \varphi,
$$

где $\varphi$-некоторая 1-форма на $M, I$ - единичный аффинор на $M$.

В карте $\left(U, x^{i}\right)$ компоненты тензорного поля $T$ кручения, удовлетворяющего условию $(1)$, представимы в виде

где $\delta_{j}^{i}$ - символ Кронекера.

$$
T_{j k}^{i}=\delta_{j}^{i} T_{k}-\delta_{k}^{i} T_{j}
$$

Необходимым и достаточным условием того, чтобы пространство линейной связности с кручением было пространством с полусимметрической линейной связностью, является выполнение в каждой системе координат равенств (см. [1]):

$$
T_{j k}^{i}=0, \quad i \neq j, k .
$$

Определение 2 (см. [1]). Векторное поле $X$ называется инфинитезимальным аффинным преобразованием связности $\nabla$, если

$$
L_{X} \nabla=0
$$

где $L_{X} \nabla$ - тензорное поле типа $(1,2)$, называемое производной Ли и задаваемое тождеством

$$
L_{X} \nabla(Y, Z)=L_{X}\left(\nabla_{Y} Z\right)-\nabla_{Y}\left(L_{X} Z\right)-\nabla_{[X, Y]} Z \text {. }
$$

Известно, что если $X$ - инфинитезимальное аффинное преобразование связности $\nabla$, то выполняются следующие условия

$$
L_{X} \nabla^{m} R=0, \quad L_{X} \nabla^{m} T=0 \quad m=0,1,2, \ldots,
$$

где $\nabla^{m} R, \nabla^{m} T$ - ковариантные дифференциалы тензорных полей кривизны $R$ и кручения $T$ связности $\nabla$. Здесь $\nabla^{0} R=R, \nabla^{0} T=T$. 
Исходя из определения линейных связностей $\hat{\nabla}, \stackrel{0}{\nabla}$, можно доказать, что уравнение $L_{X} \nabla=0$ равносильно системе уравнений

$$
L_{X} \stackrel{0}{\nabla}=0, \quad L_{X} T=0 .
$$

Тогда равенства (3) оказываются равносильными для связности $\stackrel{0}{\nabla}$ следующим соотношениям:

$$
L_{X} \stackrel{0}{\nabla^{m}} \stackrel{0}{R}=0, \quad L_{X} \nabla^{m} T=0,
$$

где $\stackrel{0}{R}$ - тензорное поле кривизны связности $\stackrel{0}{\nabla}$.

Соотношения (5) называются условиями интегрируемости уравнения $L_{X} \nabla=0$. При $m=0$ соответствующая система представляет собой первую серию условий интегрируемости (см. [1]).

3. Касательные расслоения. Для связного дифференцируемого многообразия $M$ обозначим через $T M$ его касательное расслоение. Атлас естественной гладкой структуры на $T M$ состоит из карт $\left(\pi^{-1}(U), x_{0}^{i}, x_{1}^{i}\right)$. Приведем определения лифтов некоторых объектов, которые нам понадобятся в дальнейшем. Все рассматриваемые объекты предполагаются гладкими класса $C^{\infty}$. Для любой функции $f \in C^{\infty}(M)$ функция $f_{(0)}=f \circ \pi$, где $\pi: T M \rightarrow M$-каноническая проекция, называется вертикальным лифтом, а функция $f_{(1)}=\left(\partial_{j} f\right)_{(0)} x_{1}^{j}$ - полным лифтом функции $f$ с многообразия $M$ в его касательное расслоение $T M$.

Если на многообразии $M$ задано векторное поле $X \in \mathfrak{T}_{0}^{1}(M)$, то на касательном расслоении $T M$ существуют векторные поля $X^{(1)}$ и $X^{(0)}$, называемые вертикальным и полным лифтами векторного поля $X$, соответственно, и в локальных координатах имеющие вид

$$
X^{(1)}=\left(X^{i}\right)_{(0)} \partial_{i}^{1}, \quad X^{(0)}=\left(X^{i}\right)_{(0)} \partial_{i}^{0}+\left(\partial_{j} X^{i}\right)_{(0)} x_{1}^{j} \partial_{i}^{1}
$$

(см. [7]).

Определение 3 (см. [4]). Полным лифтом линейной связности $\nabla$, заданной на многообразии $M$, называется единственная линейная связность $\nabla^{(0)}$ на $T M$, удовлетворяющая условиям

$$
\nabla_{X^{(0)}}^{(0)} Y^{(0)}=\left(\nabla_{X} Y\right)^{(0)}, \quad \nabla_{X^{(0)}}^{(0)} Y^{(1)}=\nabla_{X^{(1)}}^{(0)} Y^{(0)}=\left(\nabla_{X} Y\right)^{(1)}, \quad \nabla_{X^{(1)}}^{(0)} Y^{(1)}=0,
$$

где $X, Y \in \mathfrak{T}_{0}^{1}(M)$.

В локальной системе координат $\left(x_{0}^{i}, x_{1}^{i}\right)$ компоненты полного лифта линейной связности $\nabla^{(0)}$ будут следующие:

$$
\Gamma_{i j 0}^{00 k}=\left(\Gamma_{i j}^{k}\right)_{(0)}, \quad \Gamma_{i j 1}^{00 k}=\left(\Gamma_{i j}^{k}\right)_{(1)}, \quad \Gamma_{i j 1}^{10 k}=\left(\Gamma_{i j}^{k}\right)_{(0)}, \quad \Gamma_{i j 1}^{01 k}=\left(\Gamma_{i j}^{k}\right)_{(0)},
$$

все остальные коэффициенты связности $\nabla^{(0)}$ равны нулю.

Составляющие тензорных полей кручения $T^{(0)}$ и кривизны ${ }_{R}^{(0)}$ связности $\nabla^{(0)}$ относительно естественного подвижного репера $\left(\partial_{i}^{\alpha}\right)(\alpha=0,1)$ будут иметь вид:

$$
\begin{gathered}
T_{i j 0}^{00 k}=\left(T_{i j}^{k}\right)_{(0)}, \quad T_{i j 1}^{10 k}=\left(T_{i j}^{k}\right)_{(0)}, \quad T_{i j 1}^{01 k}=\left(T_{i j}^{k}\right)_{(0)}, \quad T_{i j 1}^{00 k}=\left(T_{i j}^{k}\right)_{(1)}, \\
\stackrel{0}{R_{l j k 0}^{000 i}}=\left(\stackrel{0}{R_{l j k}^{i}}\right)_{(0)}, \quad \stackrel{0}{R_{l j k 1}^{000 i}}=\left(\stackrel{0}{R_{l j k}^{i}}\right)_{(1)}, \quad \stackrel{0}{R_{l j k 1}^{100 i}}=\left(\stackrel{0}{R_{l j k}^{i}}\right)_{(0)}, \\
\stackrel{0}{R_{l j k 1}^{010 i}}=\left(\stackrel{0}{R_{l j k}^{i}}\right)_{(0)}, \quad \stackrel{0}{R_{l j k 1}^{001 i}}=\left(\stackrel{0}{R_{l j k}^{i}}\right)_{(0)},
\end{gathered}
$$

остальные компоненты равны нулю. 
4. Инфинитезимальные аффинные преобразования касательных расслоений полного лифта полусимметрической связности. В этом разделе будем исследовать инфинитезимальные аффинные преобразования касательного расслоения $T M$, снабженного связностью $\nabla^{(0)}$ полного лифта полусимметрической связности $\nabla$, заданной на многообразии $M$.

В координатной форме уравнение $L_{\tilde{X}} \nabla^{(0)}=0$ равносильно системе дифференциальных уравнений в частных производных второго порядка

$$
\partial_{j}^{\alpha} \partial_{k}^{\beta} X_{\sigma}^{i}+\Gamma_{m k \sigma}^{\tau \beta i} \partial_{j}^{\alpha} X_{\tau}^{m}+\Gamma_{j m \sigma}^{\alpha \tau i} \partial_{k}^{\beta} X_{\tau}^{m}-\Gamma_{j k \tau}^{\alpha \beta m} \partial_{m}^{\tau} X_{\sigma}^{i}+X_{\tau}^{m} \partial_{m}^{\tau} \Gamma_{j k \sigma}^{\alpha \beta i}=0
$$

Здесь $X_{\alpha}^{i}$ - координаты векторного поля $\tilde{X}=X_{\alpha}^{i} \partial_{i}^{\alpha}$.

Систему (6) можно представить в виде системы линейных дифференциальных уравнений первого порядка, введя новые неизвестные и полагая $A_{k \sigma}^{\alpha i}=\partial_{k}^{\alpha} X_{\sigma}^{i}$ :

$$
\partial_{j}^{\alpha} A_{k \sigma}^{\beta i}+\Gamma_{m k \sigma}^{\tau \beta i} A_{j \tau}^{\alpha m}+\Gamma_{j m \sigma}^{\alpha \tau i} A_{k \tau}^{\beta m}-\Gamma_{j k \tau}^{\alpha \beta m} A_{m \sigma}^{\tau i}+X_{\tau}^{m} \partial_{m}^{\tau} \Gamma_{j k \sigma}^{\alpha \beta i}=0 .
$$

Первую серию условий интегрируемости системы (7) представляют собой соотношения

$$
L_{\tilde{X}} T^{(0)}=0, \quad L_{\tilde{X}}{ }^{0}(0)=0, \quad k=0,1, \ldots
$$

Для оценки сверху размерностей алгебр Ли инфинитезимальных аффинных преобразований пространства $\left(T M, \nabla^{(0)}\right)$ мы ограничимся исследованием условия $L_{\tilde{X}} T^{(0)}=0$. В локальных координатах это равенство равносильно системе

$$
T_{m k \sigma}^{\tau \beta i} A_{j \tau}^{\alpha m}+T_{j m \sigma}^{\alpha \tau i} A_{k \tau}^{\beta m}-T_{j k \tau}^{\alpha \beta m} A_{m \sigma}^{\tau i}+X_{\tau}^{m} \partial_{m}^{\tau} T_{j k \sigma}^{\alpha \beta i}=0 .
$$

Систему (8) мы рассмотрим, учитывая структуру инфинитезимального аффинного преобразования $\tilde{X}$.

Так как линейная связность $\stackrel{0}{\nabla}$ не имеет кручения, то воспользуемся результатами Х. Шадыева (см. [6]), что векторное поле $\tilde{X}$ в пространстве $(T M, \stackrel{0}{\nabla}(0))$ является инфинитезимальным аффинным преобразованием тогда и только тогда, когда выполняются следующие условия:

(1) существуют векторные поля $X, Y$, тензорные поля $G, F$ типа $(1,1)$ на $M$ такие, что

$$
\tilde{X}=X^{(0)}+Y^{(1)}+G^{V \gamma}+F^{H \gamma},
$$

где

$$
G^{V \gamma}=\left(G_{i}^{j}\right)_{(0)} x_{1}^{i} \partial_{j}^{1}, \quad F^{H \gamma}=\left(F_{m}^{r}\right)_{(0)} x_{1}^{m}\left(\partial_{r}^{0}-\left(\Gamma_{r j}^{k}\right)_{(0)} x_{1}^{j} \partial_{k}^{1}\right)
$$

(2) имеют место равенства

$$
\begin{aligned}
& L_{X} \stackrel{0}{\nabla}=0, \quad L_{Y} \stackrel{0}{\nabla}=0 ; \quad \stackrel{0}{\nabla} G=0, \quad \stackrel{0}{\nabla} F=0 ; \\
& \stackrel{0}{R_{m k l}^{i}} F_{j}^{m}=F_{m}^{i} \stackrel{0}{R_{j k l}^{m}}=0 ; \quad \stackrel{0}{R_{m k l}^{i}} G_{j}^{m}-G_{m}^{i} \stackrel{0}{R_{j k l}^{m}}=0 .
\end{aligned}
$$

Представив систему (8) в развернутой форме (индексы $\alpha, \beta, \sigma$ могут принимать только два значения: 0 или 1) и подставив в получившиеся уравнения разложение (9), приходим к следующей системе уравнений:

$$
\begin{gathered}
L_{X} T=0, \quad L_{Y} T=0, \\
T_{j k}^{m} F_{m}^{i}=T_{m k}^{i} F_{j}^{m}=T_{j m}^{i} F_{k}^{m}=0, \\
T_{m k}^{i} G_{j}^{m}=T_{j m}^{i} G_{k}^{m}=T_{j k}^{m} G_{m}^{i} .
\end{gathered}
$$

Совокупность всевозможных инфинитезимальных аффинных преобразований образует алгебру Ли относительно естественных операций сложения и умножения на скаляр из $\mathbb{R}$ и операции коммутирования. Обозначив через $\tilde{L}$ алгебру Ли векторных полей вида $(9)$, а через $L^{p}$ $(p=0,1,2,3)$ - множество векторных полей вида $X^{(0)}, Y^{(1)}, G^{V \gamma}, F^{H \gamma}$, можно показать, что подпространства $L^{p}, p=0,1,2,3$, являются подалгебрами алгебры Ли $\tilde{L}$ и

$$
\operatorname{dim} \tilde{L}=\operatorname{dim} L^{0}+\operatorname{dim} L^{1}+\operatorname{dim} L^{2}+\operatorname{dim} L^{3},
$$


причем размерности подалгебр $L^{0}$ и $L^{1}$ равны размерности алгебры Ли инфинитезимальных аффинных преобразований пространства $(M, \stackrel{0}{\nabla})$ (см. [5]). Таким образом, чтобы исследовать размерность алгебры Ли $\tilde{L}$, необходимо и достаточно оценить размерность каждой из подалгебр $L^{p}$, $p=0,1,2,3$.

Оценим размерность подалгебр $L^{0}$ и $L^{1}$. И. П. Егоровым доказано, что пространства полусимметрической линейной связности с нулевым или ненулевым тензорным полем кривизны $R$ и ненулевым тензорным полем кручения $T$ имеют максимальную размерность $n^{2}$ (см. [1]). Для доказательства точности оценки рассматривается пространство $\left(\mathbb{R}^{n}, \nabla\right)$, где линейная связность $\nabla$ определяется следующими коэффициентами

$$
\begin{aligned}
& \Gamma_{1 n}^{1}=\Gamma_{2 n}^{2}=\ldots=\Gamma_{n-1 n}^{n-1}=a+b, \\
& \Gamma_{n 1}^{1}=\Gamma_{n 2}^{2}=\ldots=\Gamma_{n n-1}^{n-1}=a-b, \\
& \Gamma_{n n}^{n}=2 a,
\end{aligned}
$$

другие $\Gamma_{j k}^{i}=0$. Инфинитезимальные аффинные преобразования данного пространства будут иметь вид

$$
\partial_{1}, \partial_{2}, \ldots, \partial_{n}, x^{i} \partial_{j}, \quad i, j=1,2, \ldots, n-1, \quad x^{n} \partial_{1}, x^{n} \partial_{2}, \ldots, x^{n} \partial_{n-1} .
$$

Отсюда следует, что $\operatorname{dim} L^{0}+\operatorname{dim} L^{1}=2 n^{2}$. Базис подалгебры $L^{0}$ составляют векторные поля:

$$
\begin{gathered}
\partial_{1}^{0}, \partial_{2}^{0}, \ldots, \partial_{n}^{0}, x_{0}^{i} \partial_{j}^{0}+x_{1}^{i} \partial_{j}^{1}, \quad i, j=1,2, \ldots, n-1, \\
x_{0}^{n} \partial_{1}^{0}+x_{1}^{n} \partial_{1}^{1}, x_{0}^{n} \partial_{2}^{0}+x_{1}^{n} \partial_{2}^{1}, \ldots, x_{0}^{n} \partial_{n-1}^{0}+x_{1}^{n} \partial_{n-1}^{1},
\end{gathered}
$$

а базисом подалгебры $L^{1}$ являются векторные поля

$$
\partial_{1}^{1}, \partial_{2}^{1}, \ldots, \partial_{n}^{1}, x_{0}^{i} \partial_{j}^{1}, \quad i, j=1,2, \ldots, n-1, \quad x_{0}^{n} \partial_{1}^{1}, x_{0}^{n} \partial_{2}^{1}, \ldots, x_{0}^{n} \partial_{n-1}^{1} .
$$

Оценим размерность подалгебры $L^{2}$. Для этого рассмотрим пространство решений уравнения $\stackrel{0}{\nabla} G=0$, где $G \in \mathfrak{T}_{1}^{1}(M)$, равносильное в локальных координатах следующей системе дифференциальных уравнений первого порядка

$$
\partial_{j} G_{k}^{i}-G_{p}^{i} \Gamma_{k j}^{p}+G_{k}^{p} \Gamma_{p j}^{i}=0 .
$$

Компоненты $G_{k}^{i}$ тензорного поля $G$ удовлетворяют условиям

$$
G_{k}^{m} T_{m j}^{i}-G_{m}^{i} T_{k j}^{m}=0 .
$$

Учитывая строение тензорного поля кручения $T$ пространств полусимметрической связности, приходим к соотношениям

$$
G_{k}^{m}\left(\delta_{m}^{i} T_{j}-\delta_{j}^{i} T_{m}\right)-G_{m}^{i}\left(\delta_{k}^{m} T_{j}-\delta_{j}^{m} T_{k}\right)=0
$$

или, раскрыв скобки, к соотношениям

$$
G_{j}^{i} T_{k}-G_{k}^{m} \delta_{j}^{i} T_{m}=0 .
$$

Для аффинора $G$ справедливы равенства

$$
G_{j}^{m} T_{k m}^{i}-G_{m}^{i} T_{k j}^{m}=0 .
$$

С учетом равенств (2) получаем следующие соотношения:

$$
\delta_{k}^{i} G_{j}^{m} T_{m}-G_{k}^{i} T_{j}=0 .
$$

Свернув формулы (13) по индексам $i$ и $k$ и обозначив $g=G_{m}^{m}$, приходим к равенствам

$$
n G_{j}^{m} T_{m}-g T_{j}=0,
$$

откуда

$$
G_{j}^{m} T_{m}=\frac{1}{n} g T_{j}
$$


Из равенств (14) и (12) следует, что

$$
\left(\frac{1}{n} \delta_{j}^{i} g-G_{j}^{i}\right) T_{k}=0
$$

Так как для тензорного поля $T$ найдется компонента $T_{k} \neq 0$, то будем иметь

$$
G_{j}^{i}=\frac{1}{n} g \delta_{j}^{i}
$$

Подставив найденные компоненты $G_{j}^{i}$ в уравнения $(11)$, получим, что $\partial_{k} g=0$, следовательно, $g-$ произвольная постоянная. Таким образом, размерность пространства решений уравнения $\stackrel{0}{\nabla} G=0$ равна 1 , отсюда заключаем, что $\operatorname{dim} L^{2}=1$. Базис алгебры $L^{2}$ на $T M$ составляет векторное поле Лиувилля

$$
x_{1}^{k} \partial_{k}^{1}, \quad k=1, \ldots, n .
$$

Оценим размерность подалгебры $L^{3}$. Для аффинора $F$ справедливы равенства

$$
F_{j}^{m} T_{m k}^{i}=0, \quad F_{k}^{m} T_{j m}^{i}=0, \quad F_{m}^{i} T_{j k}^{m}=0 .
$$

Учитывая строение тензорного поля кручения $T$, соотношения (16) дают

$$
F_{j}^{i} T_{k}-F_{j}^{m} \delta_{k}^{i} T_{m}=0, \quad F_{k}^{i} T_{j}-F_{k}^{m} \delta_{j}^{i} T_{m}=0, \quad F_{j}^{i} T_{k}-F_{k}^{i} T_{j}=0 .
$$

Равенства $F_{j}^{i} T_{k}-F_{j}^{m} \delta_{k}^{i} T_{m}=0$ свернем по индексам $i$ и $k$, получим:

$$
F_{j}^{m} T_{m}-F_{j}^{m} T_{m} n=0 .
$$

Отсюда следует, что $F_{j}^{m} T_{m}=0$. Подставляя их в первую группу уравнений системы $(17)$, получим, что $F_{j}^{i} T_{k}=0$.

Поскольку $T \neq 0$, то найдется компонента $T_{k} \neq 0$, поэтому $F_{j}^{i}=0$. Следовательно, размерность пространства решений уравнения $\nabla F=0$ равна нулю, т.е. $\operatorname{dim} L^{3}=0$. Тогда

$$
\operatorname{dim} \tilde{L}=\operatorname{dim} L^{0}+\operatorname{dim} L^{1}+\operatorname{dim} L^{2}=2 n^{2}+1 .
$$

Таким образом, доказана следующая теорема.

Теорема 1. Максимальная размерность алгебр Ли инфинитезималъных афбинных преобразований касателъных расслоений со связностью полного лифта над пространствами полусимметрической линейной связности с нулевым и ненулевым тензорным полем кривизны равна $2 n^{2}+1$.

\section{СПИСОК ЛИТЕРАТУРЫ}

1. Егоров И. П. Движения в пространствах аффинной связности. - Казань: Изд-во Казанск. ун-та, 1965.

2. Егоров И. П. Геометрия. - М.: Просвещение, 1979.

3. Норден А. П. Пространства аффинной связности. - М.: Наука, 1976.

4. Султанов A. Я. Продолжения тензорных полей и связностей в расслоения Вейля// Изв. вузов. Мат. -1999 . - № 9. - C. 64-72.

5. Султанова Г. А. О некоторых подалгебрах алгебры Ли инфинитезимальных аффинных преобразований касательного расслоения $T M$ со связностью полного лифта// Сб. науч. статей Междунар. конф. «Ломоносовские чтения на Алтае: Фундаментальные проблемы науки и образования». - Барнаул: Изд-во Алтайск. ун-та, 2014. - С. 378-381.

6. Шадыев Х. Аффинная коллинеация синектической связности в касательном расслоении// Тр. геом. семин. - 1984. - 16. - С. 117-127.

7. Yano K. Tangent and Cotangent Bundles. - New York: Marcel Dekker, 1973.

Султанова Галия Алиевна

Филиал Военной Академии материально-технического обеспечения, Пенза

E-mail: sultgaliya@yandex.ru 